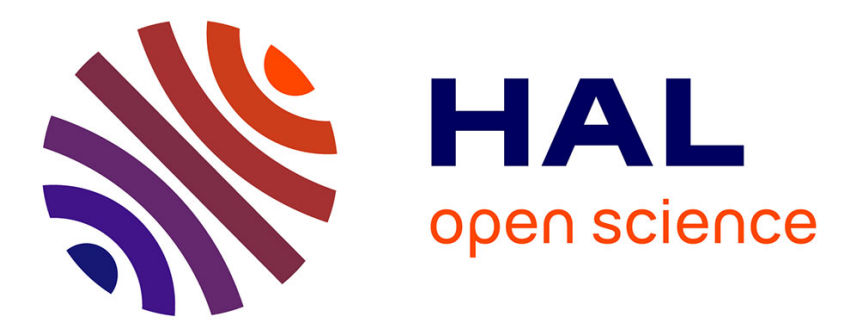

\title{
Ultrasound wave propagation in a stochastic cortical bone plate
}

S. Naili, V.-H. Nguyen, M.-B. Vu, Christophe Desceliers, Christian Soize

\section{To cite this version:}

S. Naili, V.-H. Nguyen, M.-B. Vu, Christophe Desceliers, Christian Soize. Ultrasound wave propagation in a stochastic cortical bone plate. The Fifth International Conference on Knowledge and Systems Engineering (KSE 2013), Oct 2013, Hanoi, Vietnam. pp.1-8. hal-00940383

\section{HAL Id: hal-00940383 https://hal.science/hal-00940383}

Submitted on 31 Jan 2014

HAL is a multi-disciplinary open access archive for the deposit and dissemination of scientific research documents, whether they are published or not. The documents may come from teaching and research institutions in France or abroad, or from public or private research centers.
L'archive ouverte pluridisciplinaire HAL, est destinée au dépôt et à la diffusion de documents scientifiques de niveau recherche, publiés ou non, émanant des établissements d'enseignement et de recherche français ou étrangers, des laboratoires publics ou privés. 


\title{
Ultrasound wave propagation in a stochastic cortical bone plate
}

\author{
Salah Naili ${ }^{a, b}$, Vu-Hieu Nguyen ${ }^{a, b}$, Mai-Ba $\mathrm{Vu}^{a, b}$, \\ Christophe Desceliers ${ }^{a, c}$, Christian Soize ${ }^{a, c}$ \\ ${ }^{a}$ Université Paris Est, Laboratoire Modélisation et Simulation Multi Echelle, \\ MSME UMR 8208 CNRS, \\ ${ }^{b} 61$ avenue du Général de Gaulle 94010 Créteil, France \\ ${ }^{c}$ 5, Boulevard Descartes, 77454 Marne-la-Vallée, France
}

\begin{abstract}
Ultrasonic guided-wave technologies are powerful nondestructive testing techniques to characterize bone material. This work aims to evaluate the effect due to spatial heterogeneity of bone material properties on its ultrasound response using axial transmission technique. A probabilistic model is introduced to describe the mechanical behavior of bone material. The numerical results focused on studying of FAS (First Arriving Velocity) showing that this quantity strongly depends on the dispersion induced by statistical fluctuations of stochastic elasticity field.
\end{abstract}

Keywords: random media, ultrasound wave propagation, multilayer heterogeneous medium, finite difference scheme, time domain, bone tissue

\section{Introduction}

It is well-known that cortical bone is a highly complex composite material formed by a hierarchical and multiscale constituents. Due to the fluctuation of pore distribution and physical properties of mineralization of bone tissues, cortical bone at the vascular scale is a heterogeneous and random medium. These factors would not be neglected when performing diagnostics of cortical bone. One of the most usual techniques used for diagnostics of long bone is known as ultrasonic pulsed through-transmission or axial transmission technique (ATT). This technique, which measures the wave velocity in the bone longitudinal direction, has been shown particularly suitable to predict mechanical as well as geometrical characteristics of the bone [10]. In the past, most models of ATT considered cortical long bone as a medium with homogeneous properties along its longitudinal direction. Hence, homogeneous or functionally graded material properties have been used to model the cortical bone plate $[2,7,12,13]$. In practice, the exploitation of measured signal data naturally needs to also take into account the uncertainty of material characteristics. However, most of parametric studies of wave propagation in bone are mainly limited to deterministic media.

Some studies have recently been carried out to investigate the influence of random properties of cortical bone in the context of ultrasound characterization. A probabilistic model based on the maximum entropy principle has been 
constructed for considering cortical bone plate as homogeneous or multi-layered media $[11,3,5]$. It has been shown that a simplified mechanical model with an additional stochastic modeling of bone elasticity properties are able to represent the in vivo measurements in the statistical sense. However, in these works, material inhomogeneities along longitudinal direction of bone have always been neglected.

This paper presents a stochastic model to consider the random fluctuation of material properties in both radial and longitudinal directions in a cortical bone plate. Section 2 presents the description of the problem and the numerical method to compute the time domain solution of the acoustic response of a heterogeneous bone plate coupled with fluid. Next, we provides a procedure to construct a parametric model for the elasticity properties of bone plate. Then, Section 3 shows a numerical test to study the sensitivity of $\mathrm{V}_{\mathrm{FAS}}$ due to dispersion of the stochastic elasticity field in the bone. Last, Section 4 gives some conclusions of this work.

\section{Modeling of transient wave propagation in a heterogeneous bone immersed in fluid}

\subsection{Geometrical configuration}

Let $\mathbf{R}\left(\mathrm{O} ; \mathbf{e}_{1}, \mathbf{e}_{2}, \mathbf{e}_{3}\right)$ be a reference Cartesian frame where $\left(\mathbf{e}_{1}, \mathbf{e}_{2}, \mathbf{e}_{3}\right)$ is an orthonormal basis. Figure 1 shows a common configuration for modeling an ultrasound axial transmission test. It consists of an anisotropic elastic layer sandwiched between two idealized acoustic fluids. The bone material is assumed to be heterogeneous in the plane $\left(\mathbf{e}_{1}, \mathbf{e}_{2}\right)$ but homogeneous along $\mathbf{e}_{3}$ direction. An acoustic line source, which is parallel to $\mathbf{e}_{3}$, produces an excitation at $\mathbf{x}^{s}=\left(x_{1}^{s}, x_{2}^{s}\right)$ inside the upper fluid layer. Hence, the problem may be reduced into a two-dimensional plane strain problem in the $\left(O ; \mathbf{e}_{1}, \mathbf{e}_{2}\right)$ plane. In Figure 1 , the infinite bone layer occupying the domain $\Omega^{b}$ with a constant thickness $h$ $\left(\Omega^{b}=\left\{x_{1} \in \mathbb{R}, 0 \geq x_{2} \geq-h\right\}\right)$. This bone plate is loaded on its upper and lower surfaces by two fluid halfspaces which represents the soft tissues. The upper fluid domain is denoted by $\Omega_{1}^{f}\left(\Omega_{1}^{f}=\left\{x_{1} \in \mathbb{R}, x_{2} \geq 0\right\}\right)$ and the lower one is denoted by $\Omega_{2}^{f}\left(\Omega_{2}^{f}=\left\{x_{1} \in \mathbb{R}, x_{2} \leq-h\right\}\right)$. The interfaces between the bone $\left(\Omega^{b}\right)$ and the fluids $\left(\Omega_{1}^{f}\right.$ and $\left.\Omega_{2}^{f}\right)$ are denoted by $\Gamma_{1}^{b f}$ and $\Gamma_{2}^{b f}$, respectively (see Fig. 1). In that follows, the whole of domain is denoted by $\Omega=\Omega_{1}^{f} \cup \Omega^{b} \cup \Omega_{2}^{f}$.

\subsection{Governing equations and numerical methods}

For the sake of simplifying, the described trilayer system, which consists of a heterogeneous anisotropic elastic layer $\Omega^{b}$ and two homogeneous inviscid acoustic fluid layers, will be all modeled as an elastic medium. At each point $\mathbf{x}$ in $\Omega$, the constitutive law equation is expressed by

$$
\boldsymbol{\sigma}=\overline{\mathbb{C}} \boldsymbol{\epsilon}, \quad \text { with } \boldsymbol{\epsilon}=\frac{1}{2}\left(\operatorname{grad} \mathbf{u}+(\operatorname{grad} \mathbf{u})^{T}\right),
$$




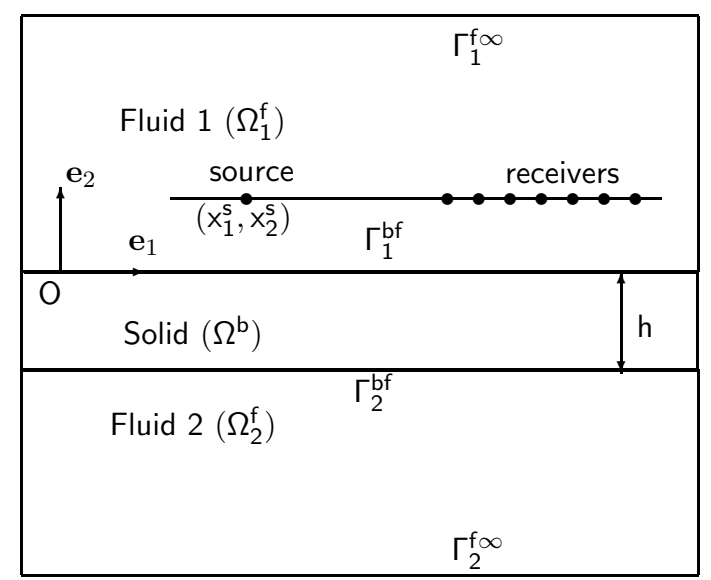

Fig. 1. Geometrical configuration of the trilayer model for ultrasound axial transmission test

where $\boldsymbol{\sigma}(\mathbf{x}, t)$ and $\boldsymbol{\epsilon}(\mathbf{x}, t)$ denote the stress and strain tensors, respectively; $\mathbf{u}(\mathbf{x}, t)$ denotes the displacement vector; $\mathbb{C}(\mathbf{x})$ is the fourth-order elasticity tensor of the fluid or solid depending on $\mathbf{x}: \overline{\mathbb{C}}(\mathbf{x})=\mathbb{C}^{f}$ for $\mathbf{x} \in \Omega_{1}^{f} \cup \Omega_{2}^{f}$ and $\overline{\mathbb{C}}(\mathbf{x})=\mathbb{C}(\mathbf{x})$ for $\mathbf{x} \in \Omega^{b}$. The elasticity tensor of the fluid $\mathbb{C}^{f}$, which is homogeneous in $\Omega_{1}^{f} \cup \Omega_{2}^{f}$, is as an isotropic elastic tensor without shear modulus. The elasticity tensor of the solid $\mathbb{C}$ depends on $\mathbf{x}$ and is determined by 6 elastic constants. Note that in the next section, the probabilistic model only concerns the tensor of elasticity of the domain $\Omega^{b}$.

By neglecting the body force field, the equation of motion in domain $\Omega$ reads

$$
\operatorname{div} \boldsymbol{\sigma}=\rho \ddot{\mathbf{u}}, \quad \forall \mathbf{x} \in \Omega,
$$

where $\rho(\mathbf{x})$ is the mass density and div designates the divergence operator. The domain $\Omega$ is at rest for $t<0$. Note that no interface condition is required for this elastoacoustic problem because both of fluid and bone domains are modeled as elastic media.

An explicit time domain finite difference scheme, which is based on a staggered grid formulation for the velocity and stress components, has been implemented [9] to solve the two-dimensional problem (2). The scheme is second-order in time and fourth-order in space. In order to avoid the wave reflected due to the boundaries at finite distances, the PML Perfectly Matched Layer (PM) technique has been is used.

The acoustic response will be captured at a linear array of receivers as shown in Fig. 1. This emitter and receiver configuration is typical one in ultrasonic axial transmission devices for characterizing the cortical layer of bone. When using the axial transmission technique, the earliest event or wavelet (also called by First Arriving Signal, FAS) of the multicomponent signal recorded at the receivers 
has been the most often investigated because it is considered as a relevant index of bone status $[18,1,8]$. In this work, the quantity of interest to be studied will be the FAS velocity.

\subsection{Construction of a parametric probabilistic model for an anisotropic elastic medium}

Parametric probabilistic models are built by modeling the local physical properties of the medium. In the present study, only the uncertainty of elasticity tensor $\mathbb{C}(\mathbf{x})$, of which the mean value is defined by $\mathbb{C}(\mathbf{x})$, will be considered. At each point $\mathbf{x} \in \Omega^{b}$, tensor $\mathbb{C}$ depends on 21 independent random variables. In practice, it is impossible to have a sufficient large set of experimental data, especially for bone material, to estimate the probability distribution of the random elasticity tensor. Moreover, the random spatial variation of the elastic property should be considered. To overcome this difficulty, we use Soize's model whereby the probability distribution is built by full-filling the maximum entropy principle. Using this principle, the stochastic elasticity tensor $\mathbb{C}(\mathbf{x})$ may be parameterized by its mean values $\mathbb{C}(\mathbf{x})$ via its matrix representation, and by a minimal set of essential parameters which consists of only 4 parameters: one scalar dispersion level $\delta$ and one vector $\boldsymbol{\lambda}$ that contains three spatial correlation lengths. The detail of essential steps to estimate the random field $\mathbb{C}(\mathbf{x} ; \mathbb{C}, \delta, \boldsymbol{\lambda})$ at every point $\mathbf{x} \in \Omega^{b}$ may be found in $[16,17]$.

\section{$3 \quad$ Numerical results}

This section will present some illustrative results on studying the ultrasound wave propagation through a random $4 \mathrm{~mm}$-thickness cortical bone layer. For this simulation, the pressure source acts on a spatial length of $0.75 \mathrm{~mm}$ which is horizontally placed in the fluid at $2 \mathrm{~mm}$ from the upper interface of the solid layer. The excitation signal is a Gaussian with a center frequency $f_{c}=1 \mathrm{MHz}$ [4]. The responses will be calculated at 14 receivers are regularly spaced with a pitch of $0.8 \mathrm{~mm}$ and a distance of emitter to closest receiver equal to $11 \mathrm{~mm}$.

Fluid and solid material properties Both fluid layers, which represent the soft tissues and bone marrow, are considered to be an inviscid water with a mass density $\rho_{f}=1000 \mathrm{~kg} . \mathrm{m}^{-3}$ and a bulk modulus $K_{f}=2.25 \mathrm{GPa}$.

The mean model of the bone material is assumed to be a transversely isotropic medium. This behavior has been experimentally shown by different authors $[6,14,15]$ to be a realistic approximation of cortical bone properties. This example uses data given by [6] who measured the homogenized bone properties by performing tensile and torsional tests with a mechanical testing system on 18 different human femoral bone specimens. The components of the mean elasticity tensor are given by: $a_{11}=23.05 \mathrm{GPa}, a_{22}=a_{33}=15.08 \mathrm{GPa}$, $a_{12}=a_{13}=8.72 \mathrm{GPa}, a_{44}=3.3 \mathrm{GPa}, a_{55}=a_{66}=4.7 \mathrm{GPa}$. 
Parameters for the uncertain elasticity model As discussed before, this study is restricted to only consider the uncertain heterogeneous in the plane $\left(\mathbf{x}_{1}, \mathbf{x}_{2}\right)$. Thus only the dispersion $\delta$ and two correlation lengths $\left(\lambda_{1}, \lambda_{2}\right)$ need to be introduced to control the random variation of elasticity field. For this example, a correlation length $\lambda_{1}=\lambda_{2}=2 \times 10^{-4} \mathrm{~m}$, which may be seen as a typical centerto-center distance between osteons in cortical bone, is used. Two different values of the dispersion $\delta=0.1$ and $\delta=0.3$ will be investigated. Figure 2 shows a map illustrating the spatial variation of $A_{i j}$.
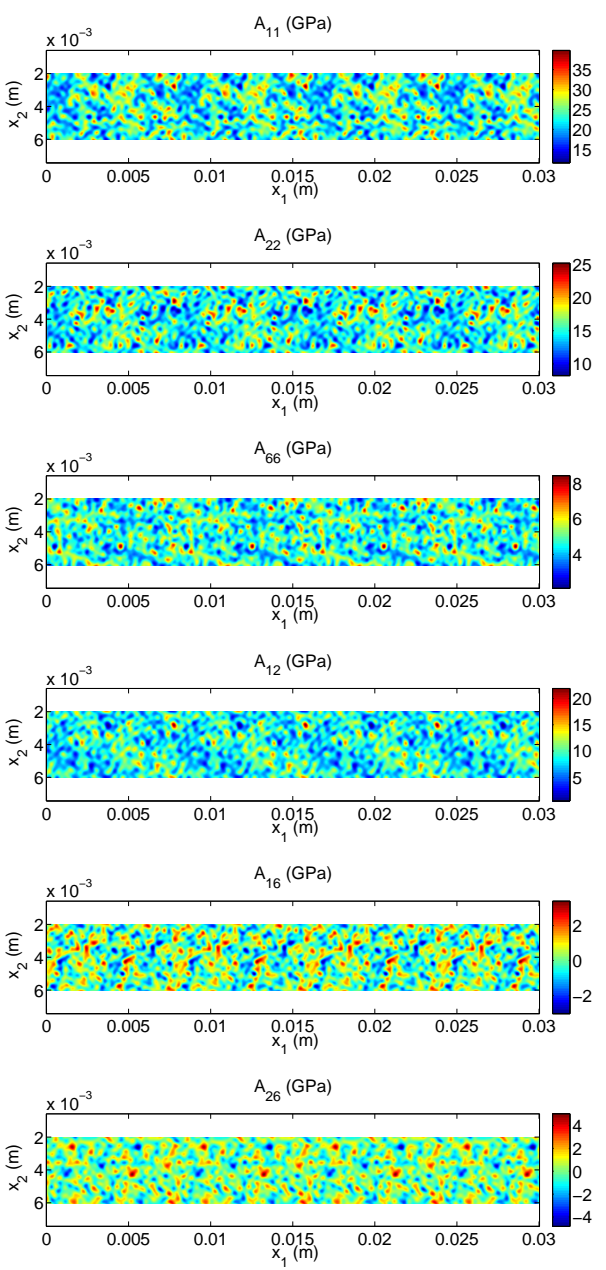

Fig. 2. Spatial variation of the elasticity tensor components $A_{i j}$ 
Numerical parameters for the finite-difference solver The finite difference simulation is performed for each of 800 Monte-Carlo realizations. A rectangular domain with size $0.03 \times 0.01 \mathrm{~m}$ is used to be able to contain all details of the model, i.e three layers with the source and receivers. The grid steps in both $x_{1}$ - and $x_{2}$-direction are chosen to be identical: $\Delta x_{1}=\Delta x_{2}=2 \times 10^{-5} \mathrm{~m}$ to ensure that grid intervals are smaller than about $1 / 8$ of the smallest wavelength in whole domains. As a result, the grid has $1501 \times 501$ points (about $1.4 \times 10^{6}$ degrees of freedom). The time step is chosen by using CFL stability condition $\Delta t<\alpha \Delta / c$, where $\alpha$ is a constant, $\Delta$ is the smallest space interval and $c$ is the maximum wave velocity in the domain. For this study, the time step size is fixed by $\Delta t=10^{-9} \mathrm{~s}$.

Results and discussions Fig. 3 (left) presents the $\mathrm{V}_{\mathrm{FAS}}$ obtained for all of 800 Monte-Carlo realizations. Two levels of dispersion $\delta=1$ and $\delta=0.3$ have been considered. It can be seen that when $\delta=0.1$, the values of $\mathrm{V}_{\mathrm{FAS}}$ oscillate around the one corresponding to the mean model which is shown as the red continuous line $\left(\mathrm{V}_{\mathrm{FAS}}^{\text {mean }}=3606 \mathrm{~m} \cdot \mathrm{s}^{-1}\right)$. When the dispersion is higher $(\delta=0.3)$, the measured values of $\mathrm{V}_{\mathrm{FAS}}$ are globally decrease. It may be explained by the fact that when the heterogeneity of the medium is greater, the scattering phenomenon becomes more significant and may modify the global wave velocity in the domain. In addition, we obtained a greater dispersion of $\mathrm{V}_{\mathrm{FAS}}$ is more important in the case $\delta=0.3$ than the one in the case $\delta=0.1$.

In Fig. 3 (right), the probability density functions of $\mathrm{V}_{\mathrm{FAS}}$ are computed for both cases. One may state that the probability density function of $V_{F A S}$ strongly depends on the dispersion $\delta$. It means that in the practice, neglecting the uncertain heterogeneity in bone may lead to a poor prediction of the mean value of mechanical properties by using $\mathrm{V}_{\mathrm{FAS}}$ as a index.
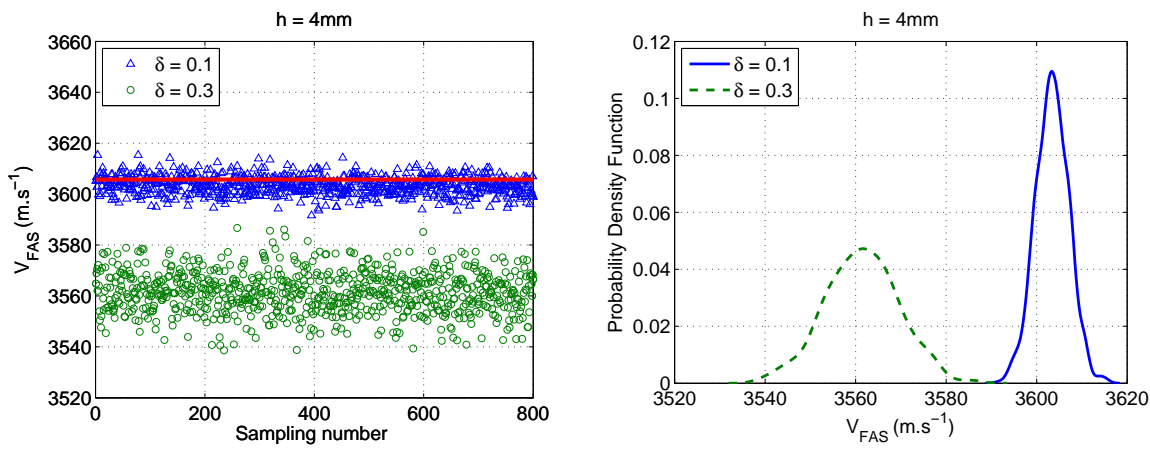

Fig. 3. (left) Values of $V_{F A S}$ evaluated at each realization; the solid line is obtained for the homogeneous material properties; (right) probability density functions of $\mathrm{V}_{\text {FAS }}$ 


\section{Conclusion}

The heterogeneity of bone's mechanical properties is significant and need to be considered when studying the sensitivity of ultrasound response in cortical long bone. As the statistical data on real bone material is hardly found, a parametric probabilistic method, which is based on the maximum entropy principle, has been used to generate an optimal probabilistic model for taking into account the uncertainties of bone elasticity. A explicit FDTD solver has been developed for simulating the wave propagation in a transversely isotropic heterogeneous medium in the time domain. It has been shown that the FAS velocity is very sensitive to dispersion of the bone's elasticity tensor in statistic sense. Detailed study need to be carried out and will be presented in a forthcoming paper.

Although the uncertain elastic model of the plate presented in this paper was developed to study behavior of cortical long bone, this procedure is of wider interest and may be applied to the characterization of other materials.

\section{References}

1. Barkmann, R., Kantorovich, E., Singal, C., Hans, D., Genant, H. K., Heller, M., and Gluer, C. C. (2000). A new method for quantitative ultrasound measurements at multiple skeletal sites. J. Clin. Densitometry, 3:1-7.

2. Bossy, E., Talmant, M., Defontaine, M., Patat, F., and Laugier, P. (2004). Bidirectional axial transmission can improve accuracy and precision of ultrasonic velocity measurement in cortical bone: a validation on test materials. IEEE Transactions on Ultrasonics, Ferroelectrics and Frequency Control, 51(1):71-79.

3. Desceliers, C., Soize, C., Grimal, M., Talmant, Q., and Naili, S. (2008a). Determination of the random anisotropic elasticity layer using transient wave propagation in a fluidsolid multilayer: model and experiments. J. Acoust. Soc. Am., 125(4):2027-2034.

4. Desceliers, C., Soize, C., Grimal, Q., Haat, G., and Naili, S. (2008b). Three dimensional transient elastic waves in multilayer semi-infinite media solved by a timespace-spectral numerical method. Wave Motion, 45(4):383-399.

5. Desceliers, C., Soize, C., Naili, S., and Haiat, G. (2012). Probabilistic model of the human cortical bone with mechanical alterations in ultrasonic range. Mechanical Systems and Signal Processing, 32:170-177.

6. Dong, X. N. and Guo, X. E. (2004). The dependence of transversely isotropic elasticity of human femoral cortical bone on porosity. J. Biomech., 37(8):1281-1287.

7. Haiat, G., Naili, S., Grimal, Q., Talmant, M., Desceliers, C., and Soize, C. (2009). Influence of a gradient of material properties on ultrasonic wave propagation in cortical bone: Application to axial transmission. J. Acoust. Soc. Am., 125(6):4043-4052.

8. Hans, D., Srivastav, S. K., Singal, C., Barkmann, R., Njeh, C. F., Kantorovich, E., Gluer, C. C., and Genant, H. K. (1999). Does combining the results from multiple bone sites measured by a new quantitative ultrasound device improve discrimination of hip fracture? J. Bone Miner. Res., 14(4):644-651.

9. Levander, A. R. (1988). Fourth-order finite-difference P-SV seismograms. Geophysics, 53(11):1425-1436.

10. Lowet, G. and Van der Perre, G. (1996). Ultrasound velocity measurements in long bones: measurement method and simulation of ultrasound wave propagation. Journal of Biomechanics, 29:1255-1262. 
11. Macocco, K., Grimal, Q., Naili, S., and Soize, C. (2006). Elastoacoustic model with uncertain mechanical properties for ultrasonic wave velocity prediction; application to cortical bone evaluation. Journal of the Acoustical Society of America, 119(2):729740 .

12. Naili, S., Vu, M.-B., Grimal, Q., Talmant, M., Desceliers, C., Soize, C., and Haiat, G. (2010). Influence of viscoelastic and viscous absorption on ultrasonic wave propagation in cortical bone: Application to axial transmission. J. AcousT. Soc. Am., 127(4):2622-2634.

13. Nguyen, V.-H. and Naili, S. (2012). Ultrasonic wave propagation in viscoelastic cortical bone plate coupled with fluids: a spectral finite element study. Computer Methods in Biomechanics and Biomedical Engineering.

14. Reilly, D. T. and Burnstein, A. H. (1974). The mechanical properties of cortical bone. J. Bone Joint Surg. Am., 56:1001-1022.

15. Rho, J. Y. (1996). An ultrasonic method for measuring the elastic properties of human tibial cortical and cancellous bone. Ultrasonics, 34(8):777-783.

16. Soize, C. (2005). Random matrix theory for modeling uncertainties in computational mechanics. Computer Methods in Applied Mechanics and Engineering, 194:1333-1366.

17. Soize, C. (2006). Non-Gaussian positive-definite matrix-valued random fields for elliptic stochastic partial differential operators. Computer Methods in Applied Mechanics and Engineering, 195:26-64.

18. Stegman, M. R., Heaney, R. P., Travers-Gustafson, D., and Leist, J. (1995). Cortical ultrasound velocity as an indicator of bone status. Osteoporos. Int., 5(5):349-533. 